\title{
Nonclinical toxicology study of recombinant-plasmid DNA anti-rabies vaccines
}

\author{
P. Uday Kumar ${ }^{\text {a }}$, B. Dinesh Kumar ${ }^{\text {a }}$, V.V. Annapurna ${ }^{\text {a }}$, T. Prasanna Krishna ${ }^{\text {a }}$, \\ S. Kalyanasundaram ${ }^{\text {a }}$, P. Suresh ${ }^{\text {a }}$, N. Harishankar ${ }^{\text {a }}$, V. Jagadeesan ${ }^{\text {a }}$, S. Hariharan ${ }^{\text {a }}$, \\ A. Nadamuni Naidu ${ }^{\text {a }}$, Kamala Krishnaswamy ${ }^{a}$, P.N. Rangarajan ${ }^{\text {b }}$, V.A. Srinivasan ${ }^{\text {c }}$, \\ G.S. Reddy ${ }^{\mathrm{c}}$, B. Sesikeran ${ }^{\mathrm{a}, *}$ \\ a National Institute of Nutrition, (Indian Council of Medical Research), Hyderabad, Andhra Pradesh 500007, India \\ ${ }^{\mathrm{b}}$ Indian Institute of Science, Bangalore, India \\ ${ }^{\mathrm{c}}$ Indian Immunologicals Limited, Hyderabad, India
}

Received 16 November 2005; received in revised form 30 December 2005; accepted 2 January 2006

Available online 13 January 2006

\begin{abstract}
The absence of standard guidelines from National and International regulatory agencies for the safety evaluation of biotechnology products challenges the ingenuity of toxicologists. At present, the development of standard pre-clinical toxicology protocols for such products is on an individual case basis. The present investigation is an attempt to evaluate the safety profile of the first indigenously developed DNA based anti-rabies vaccine in India. The test compounds were DNA rabies vaccine $\{$ DRV $(100 \mu \mathrm{g})\}$ and combination rabies vaccine $(\mathrm{CRV}(100 \mu \mathrm{g}$ DRV and 1/50 dose of cell culture vaccine)), intended for clinical use by intramuscular route on 1, 7, 14 and 28 day. As per the regular mandatory requirements, the study has been designed to undertake acute (single dose-10 days), sub-chronic (repeat dose-28 days) and chronic (intended clinical dose-120 days) toxicity tests using three dose levels viz. therapeutic, average $(2 \times$ therapeutic dose $)$ and highest dose $(10 \times$ therapeutic dose $)$ exposure in Swiss Albino mice. The selection of the rodent model viz. Swiss Albino mice is based on affinity and rapid higher antibody response during the efficacy studies. Apart from physical, physiological, clinical, hematological and histopathology profiles of all target organs, the tier-I immunotoxicity parameters have also been monitored. There were no observational adverse effects even at levels of $10 \times$ therapeutic dose administration of DRV and CRV. The procedure also emphasizes on the designing of protocols for the products developed by recombinant technique.
\end{abstract}

(C) 2006 Elsevier Ltd. All rights reserved.

Keywords: Biotech products; Toxicology; DNA rabies vaccine (DRV); Combination rabies vaccine (CRV); Abhayrab; Safety evaluation; Pre-clinical toxicology

\section{Introduction}

The safety evaluation of therapeutic agents at pre-clinical stage, especially of DNA/RNA based recombinant products is gaining momentum even in the absence of standard stipulated, regulatory guidelines [23]. In the recent past, many vaccines have been developed using modern biotechnological techniques, as they are not only potential preventive/therapeutic agents, but also economically viable

\footnotetext{
* Corresponding author. Tel.: +91 40 27008921; fax: +91 4027019074.

E-mail address: sesikerann@yahoo.com (B. Sesikeran).
}

$[11,12,18]$. Among the vaccines available in India for various diseases, the one against rabies is produced from neural tissue (sheep brain) and is reported to be associated with autoimmune neuropathy [20]. Also, the anti-rabies vaccine developed by tissue culture technique is expensive and hence unaffordable to large populations in developing countries $[10,17]$.

Based on earlier reports [24,25,1], a recombinant DNA rabies vaccine (DRV) has been developed indigenously for the first time in India by Indian Institute of Sciences (IISc), Bangalore, India. The DRV was shown to induce rabies virus neutralizing antibodies in mice and monkeys and confer 
$50-80 \%$ protection in a murine rabies virus challenge model $[5,3,4]$. However, a novel combination rabies vaccine (CRV) consisting of DRV and low doses of existing cell culture vaccine was found to be more potent since it not only induced high levels of neutralizing antibodies in mice and cattle but also conferred $100 \%$ protection in murine rabies virus challenge models [4]. As a first step towards carrying out animal and human clinical trials, the present study was undertaken to evaluate the pre-clinical toxicology profile viz. acute, subchronic and chronic toxicity test of DRV and CRV, in rodent species based on the National and International guidelines [2,14,15], FDA, [7-9].

\section{Materials and methods}

The pre-clinical toxicology data of DRV and CRV has been obtained from equal number of male and female mice as per the regulatory guidelines.

\subsection{Test formulations}

The following formulations were manufactured at Indian Immunologicals Limited, Hyderabad, India, which is approved by Indian Drug control authorities and ISO 9001 certified facility.

\subsubsection{DNA anti-rabies vaccine (DRV)}

DRV is a recombinant DNA anti-rabies vaccine comprising of plasmid DNA $(100 \mu \mathrm{g})$ encoding rabies virus surface glycoprotein in animal cells [5]. DRV plasmid DNA was prepared from transformed $E$. coli cells and purified under GMP conditions as described [13].

\subsubsection{Combination rabies vaccine (CRV)}

CRV was prepared by mixing DRV and Vero cell-derived inactivated rabies virus vaccine (Abhayrab, Indian Immunologicals Limited, India) such that $1 \times$ dose consisted of $100 \mu \mathrm{g}$ of DRV and 1/50 dose of Abhayrab. This was developed for an intended clinical dose to be administered for protection against rabies. The immunogenecity of Abhayrab has been described [19].

2.1.2.1. Test dose. DRV in various dose levels of $100(1 \times)$, $200(2 \times)$ and $1000(10 \times) \mu \mathrm{g}$ or CRV containing $1 / 50$ dose of cell culture vaccine in addition to $100,200 \mu \mathrm{g}$ DRV had been prepared as $100 \mu \mathrm{l}$ of plasmid DNA dissolved in saline, to be administered in a constant volume $(0.1 \mathrm{ml})$ to the various groups of animals, by intramuscular route under various dosage schedules using saline as a vehicle for administration (Table 1).

2.1.2.2. Test species. Swiss Albino mice (Mus Musculus), aged between 4-6 weeks, weighing 16-20 g,in equal numbers of both sexes, were obtained from National Centre for Laboratory Animal Sciences, NIN, Hyderabad, India. All the animals were conditioned to the experimental environment for a period of 3 days. Animals were housed in groups of three, in standard suspended polypropylene mice cages with top grill having facilities for holding pelleted feed and drinking water in glass bottles with stainless steel sipper tubes. The bottom of the cage was also stainless steel grilled to facilitate free droppings of faeces and urine and also to prevent coprophagy. The environmental conditions were kept at $21 \pm 2{ }^{\circ} \mathrm{C}$, with $10-15$ air changes per hour and relative humidity $50-55 \%$ with a $12 \mathrm{~h}$ light/dark cycle. The animals had free access to sterile pelleted feed of standard composition containing all macro and micronutrients. Water which was passed through activated charcoal filter and exposed to UV rays (Aqua guard on-line water filter-cum-purifier, Eureka Forbes Ltd., India) was provided. The animals were examined at regular intervals by trained personnel to look for any traits other than normal.

\section{Test details}

As mentioned earlier, all tests were undertaken as per the regulatory guidelines and all animal experimental protocols were approved by the Institutional Animal Ethics Committee before commencement of the studies.

\subsection{Acute toxicity test}

A pre-study was conducted in 10 Swiss albino mice (5 males +5 females) with the test formulation DRV containing ten times the intended clinical dose $(10 \times)$ administered as a single dose to observe morbidity, mortality and toxic reactions, if any, for 15 days.

\subsection{Sub-chronic toxicity test}

A total of 120 Swiss Albino mice ( 60 males +60 females) were equally divided randomly into 6 groups. All these animals were exposed to test compounds at various dose levels (Table 1) for three consecutive doses for 3 days. Various parameters (mentioned below) have been recorded at the mentioned intervals. The hematological, clinical chemistry, gross necropsy and histopathological parameters have been studied in 50\% of the animals on day 15 and remaining on day 30 to look for abnormal reactions, if any. The number of samples analyzed in all these studies varied from 5 to 10 .

\subsection{Chronic toxicity test}

A total of 120 Swiss Albino mice ( 60 males +60 females) were equally divided randomly into 6 groups. Each of these animals was exposed to the test formulations in accordance with an intended clinical dosage schedule viz. $0,4,7,14$ and 28 days at therapeutic, twice and 10 times of therapeutic concentrations (Table 1). Apart from various study param- 
Table 1

Test details and dosage schedules

\begin{tabular}{|c|c|c|c|c|}
\hline \multirow[t]{2}{*}{ Dose details } & \multirow[t]{2}{*}{ Test compounds } & \multicolumn{3}{|c|}{ Test details $^{\mathrm{a}}$} \\
\hline & & Pretesting & Sub-chronic & Chronic \\
\hline \multirow{2}{*}{ Therapeutic } & DRV $1 \times(100 \mu \mathrm{g})$ & & $10 \mathrm{M}+10 \mathrm{~F}$ & $10 \mathrm{M}+10 \mathrm{~F}$ \\
\hline & CRV $1 \times(100 \mu \mathrm{g}+1 / 50$ dose $)$ & - & $10 \mathrm{M}+10 \mathrm{~F}$ & $10 \mathrm{M}+10 \mathrm{~F}$ \\
\hline \multirow[t]{2}{*}{ Average } & DRV $2 \times(200 \mu \mathrm{g})$ & - & $10 \mathrm{M}+10 \mathrm{~F}$ & $10 \mathrm{M}+10 \mathrm{~F}$ \\
\hline & CRV $2 \times(200 \mu \mathrm{g}+1 / 50$ dose $)$ & - & $10 \mathrm{M}+10 \mathrm{~F}$ & $10 \mathrm{M}+10 \mathrm{~F}$ \\
\hline High dose & DRV $10 \times(1000 \mu \mathrm{g})$ & $5 \mathrm{M}+5 \mathrm{~F}$ & $10 \mathrm{M}+10 \mathrm{~F}$ & $10 \mathrm{M}+10 \mathrm{~F}$ \\
\hline Vehicle & Aluminum Hydroxide Gel (2 mg) & - & $10 \mathrm{M}+10 \mathrm{~F}$ & $10 \mathrm{M}+10 \mathrm{~F}$ \\
\hline
\end{tabular}

a Dosage schedules: $100 \mu \mathrm{l}$ of the respective test compound was administered intramuscularly as follows: pre-testing: DRV $10 \times$ single dose. Sub-chronic: exposed on three consecutive days from start (day 1), for the respective formulations. Chronic: 5 doses administered on 1, 4, 7, 14 and 28 day.

eters, hematological, biochemical profiles, gross necropsy, histopathological and immunotoxicological parameters had been studied in mice in $1 / 3$ of the animals each time on 30,60 and 120 days. The number of samples analyzed in all these studies varied from 5 to 8 .

\section{Study parameters}

\subsection{Live phase of animals}

An extensive pharmacological profile for the safety assessment of the test compound was generated (using standard international guidelines which included monitoring of food intake, body weight, general behaviour etc). Water intake was monitored twice a week and cage side observations were recorded daily.

\subsection{Physical examination}

Physical examination included observations of hair coat, lacrimation, salivation, respiration character and rate, eye prominence, eyelid closure, tremors etc. which were monitored twice a week.

\subsection{Neurological examination}

The neurological examination included locomotor, rearing activity, tail elevation, abnormal ataxic gait, head position, pinna touch response etc., which were recorded at least twice a week.

\subsection{Clinical laboratory investigations}

After the experimental period, the mice were fasted overnight (water allowed) and blood drawn from retroorbital plexus using heparanized microcapillary tubes and blood collected into heparin or EDTA K2 containing vials. Plasma was separated from heparinized blood and used for clinical chemistry analyses which included liver function tests (AST, ALT, ALP), renal function tests (urea, creatanine) in addition to plasma glucose, albumin and calcium, using $\mathrm{ACE}^{\mathrm{TM}}$ auto analyzer (Schiapparelli Biosystems, Wipro Biomed) at vari- ous time points as indicated in test details. Apart from these, qualitative tests of urine were also carried out.

\subsection{Haematological examination}

The haematological investigations included-total white blood cell (WBC) count, red blood cell (RBC) count, haemoglobin $(\mathrm{Hb})$, haematocrit (HCT), mean corpuscular volume (MCV), mean corpuscular haemoglobin $(\mathrm{MCH})$, mean corpuscular haemoglobin concentration (MCHC), platelet count, mean platelet volume (MPV) and lymphocyte \% in blood samples drawn into tubes containing EDTA $\mathrm{K} 2$ at various time points, as indicated in the test details and analysed on an automated blood cell counter (Serono Baker System $9120 \mathrm{CP}+$, UK).

\subsection{Gross necropsy and histopathology}

After collecting the blood samples, the mice were euthanised by cervical dislocation and subjected to gross necropsy. External features suggesting any abnormality, especially evidence of lymph node enlargement were looked into. After opening the chest and abdominal cavities, an in situ examination was done. Brain, heart, lungs, liver, spleen, kidneys, gastrointestinal tract, pancreas, individual sex organs, thymus, thyroid, trachea, adrenals, bone marrow and injection site were studied and the findings recorded. The individual organs were again examined for gross morphology changes after removal. After detailed gross necropsy examination, liver, spleen, kidney, lung, heart, brain, ovaries/testes were weighed on a top loading electronic weighing machine (Sartorious, US). They were adequately sliced and preserved in $10 \%$ neutral buffered formalin overnight, sampled and processed by conventional methods. Five micron paraffin sections were stained with Hematoxylin and Eosin and examined under a light microscope (Leitz Diaplan, USA). All deviations from normal histology were recorded and compared with corresponding controls.

\section{Immunotoxicology (immunopathology)}

The tier-I parameters include clinical (hair loss, salivation, lacrimation, skin reaction at injection site), heamatological 


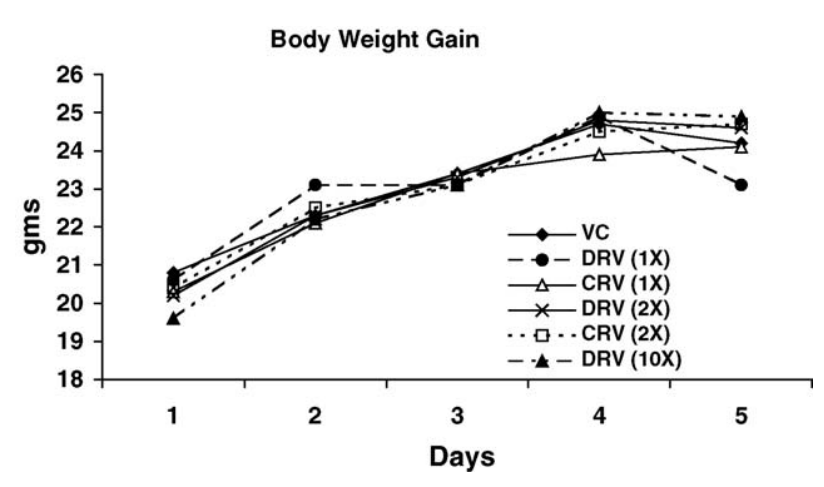

Fig. 1. Sub-chronic-mice. $1=$ Baseline; $2=11$ th Day (10th day after last exposure); $3=15$ th day after last exposure; $4=25$ th day after last exposure; $5=30$ th day after last exposure.

(total leucocyte count and differential leucocyte counts) as well as histopathological studies for immunotoxicology evaluation. The histological evidence of any immune mediated hyperactivity or immune suppression were in the form of reactive hyperplasia/hypoplasia at the injection site, spleen, thymus, mucosa associated lymphoid tissue, bone marrow etc [21].

\section{Statistical analysis}

Individual group comparisons (Fisher's Exact test, Chisquare test), Ranked Data (Mann-Whitney $U$ test, KruskalWallis one-way ANOVA, Spearman Correlation Coefficient), Paired Comparison (Wilcoxon matched-pair signed rank test), Continuous Data (Levene's/Bartlett test, one-way ANOVA, Dunnett's Post Hoc test, Pearson's Correlation Coefficient), Paired Comparison (Matched Paired $t$ test) were employed.

\section{Results}

\subsection{Pre-testing}

The mice exposed to the single dose of ten times the intended clinical dose of DRV did not show any abnormal behaviour/toxic signs and no lethality.

\subsection{Sub-chronic}

The mice, investigated after administration of DRV, CRV at various dose levels did not show any significant changes in body weight gains (Fig. 1) or food intakes. There were
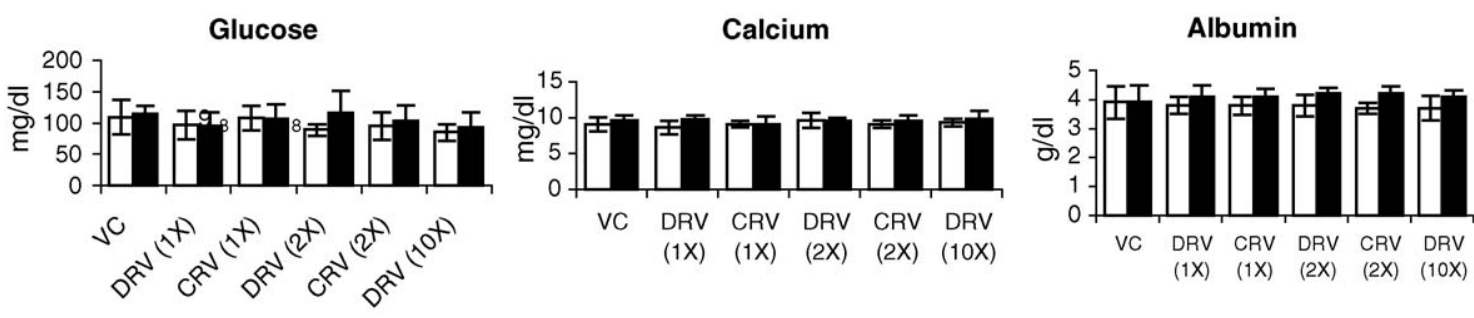

Renal Function Test
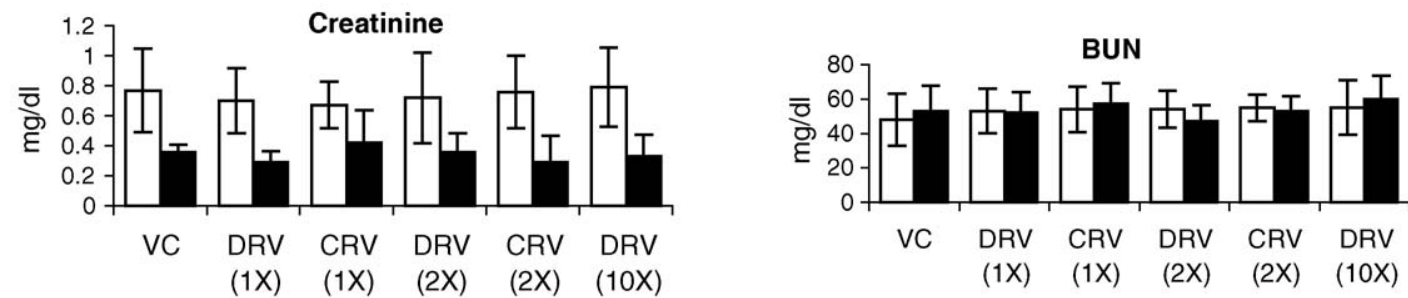

Liver Function Test
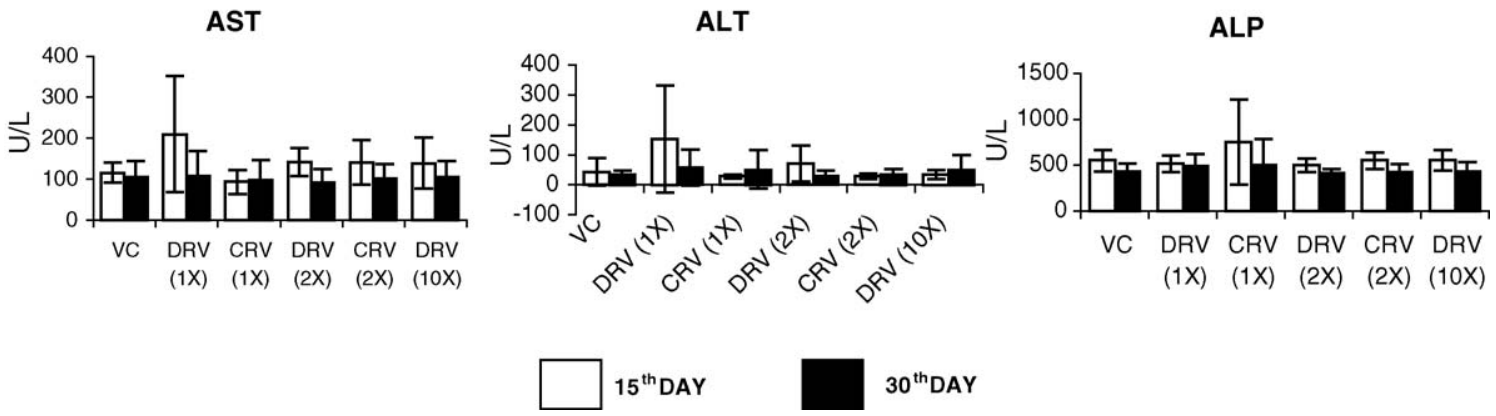

$30^{\text {th }}$ DAY

Fig. 2. Sub-chronic - mice - biochemistry. 
Table 2

Heamatological profile of the animals exposed to test compound Sub-chronic toxicity test

\begin{tabular}{|c|c|c|c|c|c|c|c|}
\hline \multirow[t]{2}{*}{ Parameters } & \multirow[t]{2}{*}{ Days } & \multicolumn{6}{|l|}{ Groups } \\
\hline & & $\mathrm{VC}$ & $\operatorname{DRV}(1 \times)$ & $\operatorname{CRV}(1 \times)$ & $\operatorname{DRV}(2 \times)$ & $\operatorname{CRV}(2 \times)$ & $\operatorname{DRV}(10 \times)$ \\
\hline $\mathrm{RBC} \times 10^{6}(\mu \mathrm{l})$ & $\begin{array}{l}15 \\
30\end{array}$ & $\begin{array}{l}10.99 \pm 0.467(10) \\
10.66 \pm 0.779(10)\end{array}$ & $\begin{array}{l}10.76 \pm 0.563(10) \\
10.57 \pm 0.505(10)\end{array}$ & $\begin{array}{l}10.72 \pm 0.449(10) \\
10.40 \pm 1.228(10)\end{array}$ & $\begin{array}{l}11.05 \pm 0.671(10) \\
10.50 \pm 1.007(10)\end{array}$ & $\begin{array}{l}10.95 \pm 0.671(10) \\
10.51 \pm 1.051(9)\end{array}$ & $\begin{array}{l}10.80 \pm 0.690(10) \\
10.52 \pm 0.281(6)\end{array}$ \\
\hline Haemoglobin (g/dl) & $\begin{array}{l}15 \\
30\end{array}$ & $\begin{array}{l}17.05 \pm 3.196(10) \\
17.99 \pm 1.065(10)\end{array}$ & $\begin{array}{l}18.21 \pm 0.870(10) \\
17.65 \pm 0.863(10)\end{array}$ & $\begin{array}{l}18.02 \pm 0.890(10) \\
17.21 \pm 1.688(10)\end{array}$ & $\begin{array}{l}18.03 \pm 1.904(10) \\
16.95 \pm 1.586(10)\end{array}$ & $\begin{array}{l}18.33 \pm 1.161(10) \\
17.27 \pm 1.538(9)\end{array}$ & $\begin{array}{l}17.84 \pm 1.152(10) \\
17.15 \pm 0.432(6)\end{array}$ \\
\hline Haematocrit (\%) & $\begin{array}{l}15 \\
30\end{array}$ & $\begin{array}{l}53.20 \pm 3.294(10) \\
51.59 \pm 4.178(10)\end{array}$ & $\begin{array}{l}54.03 \pm 5.572(10) \\
50.39 \pm 3.056(10)\end{array}$ & $\begin{array}{l}52.70 \pm 2.569(10) \\
49.94 \pm 6.243(10)\end{array}$ & $\begin{array}{l}53.49 \pm 2.767(10) \\
49.87 \pm 3.545(10)\end{array}$ & $\begin{array}{l}53.20 \pm 2.920(10) \\
50.06 \pm 5.350(9)\end{array}$ & $\begin{array}{l}52.11 \pm 3.138(10) \\
49.35 \pm 1.171(6)\end{array}$ \\
\hline $\mathrm{MCHC}(\%)$ & $\begin{array}{l}15 \\
30\end{array}$ & $\begin{array}{l}33.97 \pm 0.766(10) \\
34.57 \pm 1.026(10)\end{array}$ & $\begin{array}{l}34.39 \pm 0.740(10) \\
35.07 \pm 0.891(10)\end{array}$ & $\begin{array}{l}34.23 \pm 0.814(10) \\
35.43 \pm 2.030(10)\end{array}$ & $\begin{array}{l}34.43 \pm 1.111(10) \\
34.50 \pm 1.020(10)\end{array}$ & $\begin{array}{l}34.44 \pm 0.525(10) \\
34.62 \pm 1.317(9)\end{array}$ & $\begin{array}{l}34.35 \pm 0.711(10) \\
34.78 \pm 0.830(6)\end{array}$ \\
\hline $\mathrm{WBC} \times 10^{3}(\mu \mathrm{l})$ & $\begin{array}{l}15 \\
30\end{array}$ & $\begin{array}{l}6.48 \pm 2.639(10) \\
6.16 \pm 1.706(10)\end{array}$ & $\begin{array}{l}5.89 \pm 2.470(10) \\
6.40 \pm 1.861(10)\end{array}$ & $\begin{array}{l}5.91 \pm 1.203(10) \\
5.42 \pm 1.813(10)\end{array}$ & $\begin{array}{l}6.56 \pm 1.895(10) \\
6.45 \pm 3.033(10)\end{array}$ & $\begin{array}{l}6.23 \pm 0.983(10) \\
6.91 \pm 1.325(9)\end{array}$ & $\begin{array}{l}6.59 \pm 1.024(10) \\
8.02 \pm 1.367(6)\end{array}$ \\
\hline
\end{tabular}

Values are expressed as mean \pm S.D. () no. of animals.

Table 3

Organ weights (g) sub-chronic study

\begin{tabular}{|c|c|c|c|c|c|c|c|}
\hline \multirow[t]{2}{*}{ Tissues } & \multirow[t]{2}{*}{ Days } & \multicolumn{6}{|l|}{ Groups } \\
\hline & & VC & $\operatorname{DRV}(1 \times)$ & $\operatorname{CRV}(1 \times)$ & $\operatorname{DRV}(2 \times)$ & $\operatorname{CRV}(2 \times)$ & $\operatorname{DRV}(10 \times)$ \\
\hline Brain & $\begin{array}{l}15 \\
30\end{array}$ & $\begin{array}{l}0.39 \pm 0.032(10) \\
0.40 \pm 0.047(10)\end{array}$ & $\begin{array}{l}0.37 \pm 0.048(10) \\
0.40 \pm 0.047(10)\end{array}$ & $\begin{array}{l}0.40 \pm 0.000(10) \\
0.40 \pm 0.000(10)\end{array}$ & $\begin{array}{l}0.39 \pm 0.032(10) \\
0.40 \pm 0.082(10)\end{array}$ & $\begin{array}{l}0.39 \pm 0.032(10) \\
0.40 \pm 0.000(10)\end{array}$ & $\begin{array}{l}0.40 \pm 0.000(10) \\
0.37 \pm 0.048(10)\end{array}$ \\
\hline Heart & $\begin{array}{l}15 \\
30\end{array}$ & $\begin{array}{l}0.10 \pm 0.000(10) \\
0.11 \pm 0.032(10)\end{array}$ & $\begin{array}{l}0.10 \pm 0.000(10) \\
0.10 \pm 0.000(10)\end{array}$ & $\begin{array}{l}0.10 \pm 0.000(10) \\
0.10 \pm 0.000(10)\end{array}$ & $\begin{array}{l}0.10 \pm 0.000(10) \\
0.10 \pm 0.000(10)\end{array}$ & $\begin{array}{l}0.10 \pm 0.000(10) \\
0.10 \pm 0.000(10)\end{array}$ & $\begin{array}{l}0.10 \pm 0.000(10) \\
0.10 \pm 0.000(10)\end{array}$ \\
\hline Kidney (L+R) & $\begin{array}{l}15 \\
30\end{array}$ & $\begin{array}{l}0.33 \pm 0.082(10) \\
0.37 \pm 0.048(10)\end{array}$ & $\begin{array}{l}0.32 \pm 0.092(10) \\
0.41 \pm 0.099(10)\end{array}$ & $\begin{array}{l}0.31 \pm 0.137(10) \\
0.41 \pm 0.074(10)\end{array}$ & $\begin{array}{l}0.30 \pm 0.105(10) \\
0.40 \pm 0.163(10)\end{array}$ & $\begin{array}{l}0.31 \pm 0.099(10) \\
0.36 \pm 0.070(10)\end{array}$ & $\begin{array}{l}0.31 \pm 0.120(10) \\
0.39 \pm 0.088(10)\end{array}$ \\
\hline Liver & $\begin{array}{l}15 \\
30\end{array}$ & $\begin{array}{l}1.02 \pm 0.140(10) \\
1.08 \pm 0.114(10)\end{array}$ & $\begin{array}{l}1.00 \pm 0.133(10) \\
1.05 \pm 0.177(10)\end{array}$ & $\begin{array}{l}0.97 \pm 0.157(10) \\
1.08 \pm 0.162(10)\end{array}$ & $\begin{array}{l}0.97 \pm 0.226(10) \\
1.06 \pm 0.117(10)\end{array}$ & $\begin{array}{l}1.03 \pm 0.177(10) \\
1.05 \pm 0.085(10)\end{array}$ & $\begin{array}{l}0.98 \pm 0.204(10) \\
1.06 \pm 0.107(10)\end{array}$ \\
\hline Spleen & $\begin{array}{l}15 \\
30\end{array}$ & $\begin{array}{l}0.10 \pm 0.000(10) \\
0.10 \pm 0.000(10)\end{array}$ & $\begin{array}{l}0.10 \pm 0.000(10) \\
0.10 \pm 0.000(10)\end{array}$ & $\begin{array}{l}0.10 \pm 0.000(10) \\
0.10 \pm 0.000(10)\end{array}$ & $\begin{array}{l}0.10 \pm 0.000(10) \\
0.11 \pm 0.032(10)\end{array}$ & $\begin{array}{l}0.10 \pm 0.000(10) \\
0.10 \pm 0.000(10)\end{array}$ & $\begin{array}{l}0.10 \pm 0.000(10) \\
0.10 \pm 0.000(10)\end{array}$ \\
\hline Testis $(\mathrm{L}+\mathrm{R})$ & $\begin{array}{l}15 \\
30\end{array}$ & $\begin{array}{l}0.26 \pm 0.089(5) \\
0.24 \pm 0.055(5)\end{array}$ & $\begin{array}{l}0.20 \pm 0.000(5) \\
0.26 \pm 0.055(5)\end{array}$ & $\begin{array}{l}0.24 \pm 0.089(5) \\
0.18 \pm 0.084(5)\end{array}$ & $\begin{array}{l}0.22 \pm 0.045(5) \\
0.26 \pm 0.055(5)\end{array}$ & $\begin{array}{l}0.20 \pm 0.000(5) \\
0.28 \pm 0.045(5)\end{array}$ & $\begin{array}{l}0.20 \pm 0.000(5) \\
0.20 \pm 0.000(5)\end{array}$ \\
\hline
\end{tabular}

Values are mean \pm S.D. () No of animals. 
no significant abnormalities in physical, physiological and neurological activities as evaluated by standard procedures.

The hematological (Table 2) and biochemical (Fig. 2) parameters on 15 day and 30 days of postexposure to the test compounds were found to be within normal range. The organ weights were not significantly different (Table 3) and there were no gross necropsy or histopathological changes in all the vital organs collected on 15 and 30 day of post exposure (Table 4).

\subsection{Chronic}

The gain in body weight (Fig. 3) and food intake was found to be normal in all test groups and there were no significant differences on day 120 of the post administration of test compound at the intended clinical dose. Similarly behavioural, clinical, physical, physiological parameters were also normal during the course of study in all groups.

The hematological (Table 5) and clinical chemistry (Fig. 4) parameters were found to be in normal range during the 120 days of post exposure to test compounds. There were no significant changes in organ weights (Table 6) and no gross morphological changes. There was no evidence of abnormal histopathological findings in all the vital organs studied (Table 7).

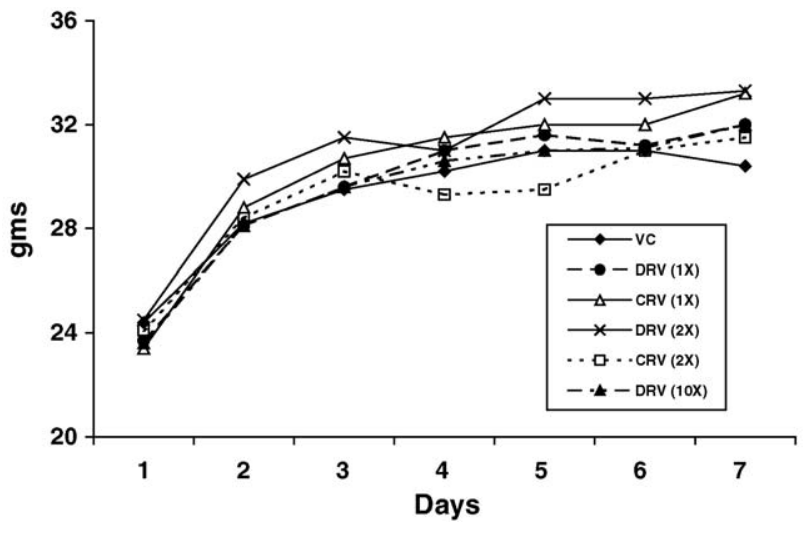

Fig. 3. Body weight gain in animals exposed to test compound-chronic toxicity test; $1=$ Before exposure; $2=29$ th day (1st day after last exposure); $3=56$ th day (30th day after last exposure); $4=79$ th day (45th day after last exposure); $5=86$ th day (60th day after last exposure); $6=100$ th day (75th day after last exposure); $7=119$ th day (90th day after last exposure).

Bio-chemical, heamatological, histopathological and immune parameters were studied for toxicological evaluation. Of these, clinical and hematology parameters were not different between the various groups studied. However in histopathological studies, it was observed that changes were scattered in all groups including vehicle control (spleen, lym-

Table 4

Gross necropsy sub-chronic study

\begin{tabular}{|c|c|c|c|c|c|c|c|c|c|c|c|c|}
\hline \multirow[b]{3}{*}{ Sex and no. of animals } & \multicolumn{12}{|c|}{ DOSAGE } \\
\hline & \multicolumn{2}{|l|}{$\mathrm{VC}$} & \multicolumn{2}{|c|}{$\operatorname{DRV}(1 \times)$} & \multicolumn{2}{|c|}{ CRV $(1 \times)$} & \multicolumn{2}{|c|}{$\operatorname{DRV}(2 \times)$} & \multicolumn{2}{|c|}{$\operatorname{CRV}(2 \times)$} & \multicolumn{2}{|c|}{ DRV $(10 \times)$} \\
\hline & M 10 & F 10 & M 10 & F 10 & M 10 & F 10 & M 10 & F 10 & M 10 & F 10 & M 10 & F 10 \\
\hline No. dead during treatment & 0 & 0 & 0 & 0 & 0 & 0 & 0 & 0 & 0 & 0 & 0 & 0 \\
\hline No. moribund and sacrificed & 0 & 0 & 0 & 0 & 0 & 0 & 0 & 0 & 0 & 0 & 0 & 0 \\
\hline No. finally sacrificed & 10 & 10 & 10 & 10 & 10 & 10 & 10 & 10 & 10 & 10 & 10 & 10 \\
\hline No. examined for gross pathology & 10 & 10 & 10 & 10 & 10 & 10 & 10 & 10 & 10 & 10 & 10 & 10 \\
\hline No. showing gross pathology & 0 & 0 & 0 & 0 & 0 & 0 & 0 & 0 & 0 & 0 & 1 & 0 \\
\hline Visceral organ pathology Kidney cyst & 0 & 0 & 0 & 0 & 0 & 0 & 0 & 0 & 0 & 0 & 1 & 0 \\
\hline
\end{tabular}

Table 5

Heamatological profile of the animals exposed to test compound Chronic toxicity test

\begin{tabular}{|c|c|c|c|c|c|c|c|}
\hline \multirow[t]{2}{*}{ Parameters } & \multirow[t]{2}{*}{ Days } & \multicolumn{6}{|l|}{ Groups } \\
\hline & & $\mathrm{VC}$ & $\operatorname{DRV}(1 \times)$ & CRV $(1 \times)$ & $\operatorname{DRV}(2 \times)$ & CRV $(2 \times)$ & $\operatorname{DRV}(10 \times)$ \\
\hline \multirow[t]{2}{*}{$\mathrm{RBC} \times 10^{6}(\mu \mathrm{l})$} & 90 & $9.79 \pm 0.592(5)$ & $9.88 \pm 0.629(5)$ & $9.48 \pm 0.254(5)$ & $9.55 \pm 0.417(5)$ & $9.83 \pm 0.189(5)$ & $9.74 \pm 0.399(5)$ \\
\hline & 120 & $8.95 \pm 1.492(5)$ & $9.78 \pm 0.420(7)$ & $9.99 \pm 0.433(6)$ & $9.63 \pm 0.661(8)$ & $10.09 \pm 0.378(7)$ & $10.18 \pm 0.355(8)$ \\
\hline \multirow[t]{2}{*}{ RDW (\%) } & 90 & $15.68 \pm 1.279(5)$ & $16.54 \pm 1.252(5)$ & $15.24 \pm 0.555(5)$ & $16.14 \pm 2.169(5)$ & $15.94 \pm 1.019(5)$ & $15.40 \pm 0.992(5)$ \\
\hline & 120 & $18.28 \pm 3.950(5)$ & $16.40 \pm 1.049(7)$ & $15.38 \pm 0.343(6)$ & $17.94 \pm 5.175(8)$ & $15.80 \pm 0.726(7)$ & $15.59 \pm 0.572(8)$ \\
\hline \multirow[t]{2}{*}{ Hemoglobing (dl) } & 90 & $15.26 \pm 0.783(5)$ & $15.78 \pm 0.638(5)$ & $15.42 \pm 0.438(5)$ & $14.26 \pm 1.613(5)$ & $15.32 \pm 0.455(5)$ & $15.58 \pm 0.522(5)$ \\
\hline & 120 & $14.54 \pm 3.031(5)$ & $15.60 \pm 0.440(7)$ & $16.15 \pm 0.345(6)$ & $14.39 \pm 2.721(8)$ & $15.84 \pm 0.321(7)$ & $16.10 \pm 0.545(8)$ \\
\hline \multirow[t]{2}{*}{ Haematocrit (\%) } & 90 & $47.44 \pm 2.2652(5)$ & $49.14 \pm 1.937(5)$ & $47.62 \pm 1.494(5)$ & $44.50 \pm 3.378(5)$ & $46.84 \pm 1.472(5)$ & $48.20 \pm 1.667(5)$ \\
\hline & 120 & $44.58 \pm 9.507(5)$ & $47.66 \pm 3.150(7)$ & $49.72 \pm 2.946(6)$ & $44.46 \pm 5.298(8)$ & $48.79 \pm 2.904(7)$ & $49.30 \pm 1.876(8)$ \\
\hline \multirow[t]{2}{*}{$\mathrm{MCHC}(\%)$} & 90 & $32.20 \pm 0.274(5)$ & $32.14 \pm 0.351(5)$ & $32.36 \pm 0.456(5)$ & $31.98 \pm 1.379(5)$ & $32.74 \pm 0.434(5)$ & $32.40 \pm 0.308(5)$ \\
\hline & 120 & $32.70 \pm 1.602(5)$ & $32.89 \pm 1.856(7)$ & $32.53 \pm 1.577(6)$ & $32.16 \pm 3.075(8)$ & $32.61 \pm 1.406(7)$ & $32.67 \pm 1.241(8)$ \\
\hline \multirow[t]{2}{*}{$\mathrm{WBC} \times 10^{3}(\mu 1)$} & 90 & $6.30 \pm 2.062(5)$ & $6.78 \pm 3.399(5)$ & $6.06 \pm 1.021(5)$ & $10.36 \pm 9.244(5)$ & $4.30 \pm 1.930(5)$ & $5.38 \pm 1.266(5)$ \\
\hline & 120 & $6.08 \pm 2.410(5)$ & $5.35 \pm 1.178(7)$ & $4.37 \pm 0.737(6)$ & $9.95 \pm 14.208(8)$ & $2.87 \pm 0.673(7)$ & $3.33 \pm 1.671(8)$ \\
\hline
\end{tabular}

Values are expressed as mean \pm S.D. () no. of animals. 

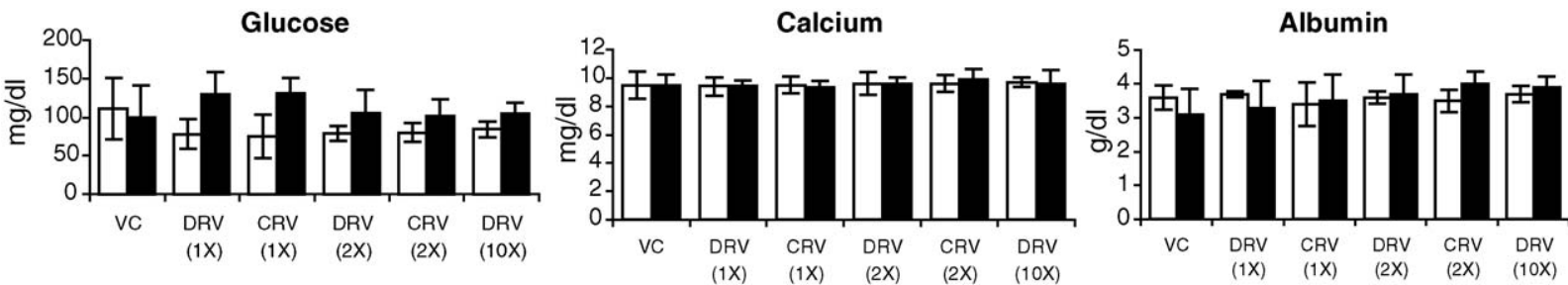

Renal Function Test
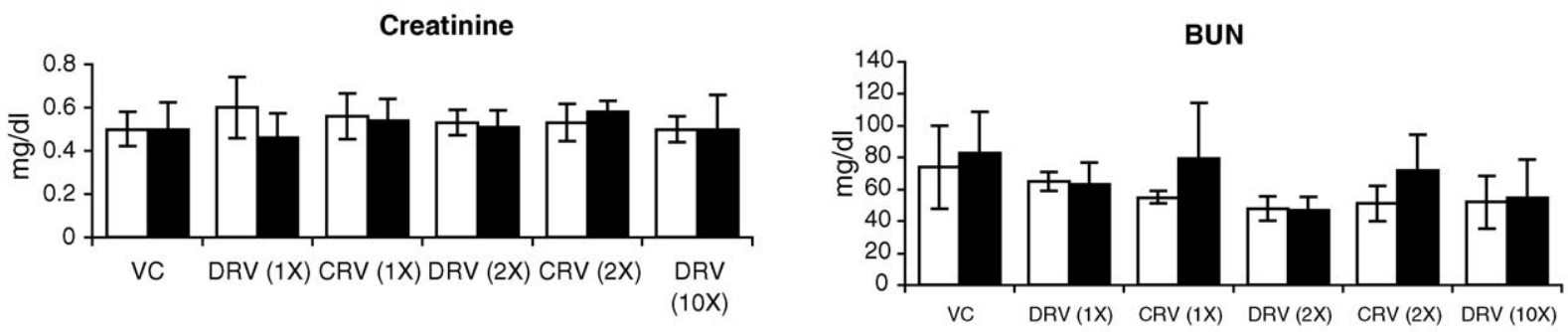

Liver FunctionTest
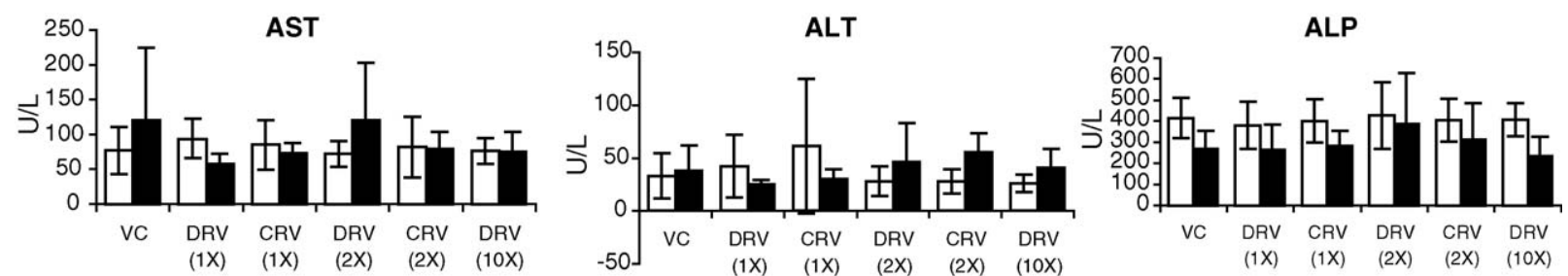

$30^{\text {th }}$ DAY

$120^{\text {th }}$ DAY

Fig. 4. Biochemistry—chronic toxicity test.

Table 6

Organ weights (g) chronic toxicity study

\begin{tabular}{lrllllll}
\hline Tissues & Days & Groups & & & & \\
\cline { 3 - 6 } & & VC & DRV $(1 \times)$ & CRV $(1 \times)$ & DRV $(2 \times)$ & CRV $(2 \times)$ & DRV $(10 \times)$ \\
\hline Brain & 60 & $0.37 \pm 0.081(6)$ & $0.33 \pm 0.103(6)$ & $0.35 \pm 0.054(6)$ & $0.33 \pm 0.051(6)$ & $0.36 \pm 0.054(5)$ & $0.35 \pm 0.055(6)$ \\
& 120 & $0.43 \pm 0.103(8)$ & $0.44 \pm 0.106(8)$ & $0.39 \pm 0.107(7)$ & $0.34 \pm 0.130(8)$ & $0.39 \pm 0.037(7)$ & $0.33 \pm 0.070(8)$ \\
Heart & 60 & $0.11 \pm 0.032(6)$ & $0.13 \pm 0.051(6)$ & $0.10 \pm(6)$ & $0.17 \pm 0.040(6)$ & $0.14 \pm 0.054(5)$ & $0.13 \pm 0.052(6)$ \\
& 120 & $0.10 \pm 0(8)$ & $0.13 \pm 0.052(8)$ & $0.11 \pm 0.037(7)$ & $0.10 \pm 0(8)$ & $0.10 \pm 0(7)$ & $0.13 \pm 0.035(8)$ \\
Kidney (L) & 60 & $0.10 \pm 0(6)$ & $0.12 \pm 0.040(6)$ & $0.12 \pm 0.040(6)$ & $0.12 \pm 0.040(6)$ & $0.10 \pm 0(5)$ & $0.12 \pm 0.040(6)$ \\
& 120 & $0.12 \pm 0.037(8)$ & $0.14 \pm 0.049(8)$ & $0.16 \pm 0.053(7)$ & $0.19 \pm 0.095(8)$ & $0.14 \pm 0.077(7)$ & $0.15 \pm 0.053(8)$ \\
Kidney (R) & 60 & $0.18 \pm 0.040(6)$ & $0.18 \pm 0.040(6)$ & $0.18 \pm 0.075(6)$ & $0.17 \pm 0.052(6)$ & $0.18 \pm 0.047(5)$ & $0.18 \pm 0.040(6)$ \\
& 120 & $0.19 \pm 0.056(8)$ & $0.23 \pm 0.059(8)$ & $0.47 \pm 0.068(7)$ & $0.26 \pm 0.157(8)$ & $0.18 \pm 0.089(7)$ & $0.19 \pm 0.099(8)$ \\
Liver & 60 & $1.27 \pm 0.150(6)$ & $1.25 \pm 0.164(6)$ & $1.37 \pm 0.082(6)$ & $1.32 \pm 0.117(6)$ & $1.26 \pm 0.055(5)$ & $1.21 \pm 0.103(6)$ \\
& 120 & $1.24 \pm 0.596(8)$ & $1.12 \pm 0.124(8)$ & $1.14 \pm 0.139(7)$ & $1.74 \pm 1.405(8)$ & $1.23 \pm 0.111(7)$ & $1.15 \pm 0.177(8)$ \\
Spleen & 60 & $0.25 \pm 0.367(6)$ & $0.10 \pm 0(6)$ & $0.12 \pm 0.040(6)$ & $0.10 \pm 0(6)$ & $0.10 \pm 0(5)$ & $0.10 \pm 0(6)$ \\
& 120 & $0.19 \pm 0.120(8)$ & $0.10 \pm 0(8)$ & $0.10 \pm 0(7)$ & $0.12 \pm 0.046(8)$ & $0.10 \pm 0(7)$ & $0.09 \pm 0.019(8)$ \\
Testis (L) & 60 & $0.13 \pm 0.057(3)$ & $0.13 \pm 0.057(3)$ & $0.10 \pm 0(3)$ & $0.10 \pm 0(3)$ & $0.15 \pm 0.070(2)$ & $0.13 \pm 0.057(3)$ \\
& 120 & $0.15 \pm 0.070(2)$ & $0.07 \pm 0.028(4)$ & $0.12 \pm 0.050(4)$ & $0.10 \pm 0(4)$ & $0.10 \pm 0(3)$ & $0.10 \pm 0(4)$ \\
Testi (R) & 60 & $0.16 \pm 0.057(3)$ & $0.13 \pm 0.057(3)$ & $0.10 \pm 0(3)$ & $0.10 \pm 0(3)$ & $0.15 \pm 0.070(2)$ & $0.13 \pm 0.057(3)$ \\
& 120 & - & $0.07 \pm 0.028(4)$ & $0.12 \pm 0.050(4)$ & $0.10 \pm 0(4)$ & $0.10 \pm 0(3)$ & $0.10 \pm 0(4)$ \\
\hline
\end{tabular}

Values are expressed as mean \pm S.D. () no. of animals. 


\begin{tabular}{|c|c|c|c|c|c|c|c|c|c|c|c|c|}
\hline \multirow[b]{3}{*}{ Sex and no. of animals } & \multicolumn{12}{|c|}{ DOSAGE } \\
\hline & \multicolumn{2}{|l|}{$\mathrm{VC}$} & \multicolumn{2}{|c|}{$\operatorname{DRV}(1 \times)$} & \multicolumn{2}{|c|}{$\operatorname{CRV}(1 \times)$} & \multicolumn{2}{|c|}{$\operatorname{DRV}(2 \times)$} & \multicolumn{2}{|c|}{$\operatorname{CRV}(2 \times)$} & \multicolumn{2}{|c|}{$\operatorname{DRV}(10 \times)$} \\
\hline & M 10 & F 10 & M 10 & F 10 & M 10 & F 10 & M 10 & F 10 & M 10 & F 10 & M 10 & F 10 \\
\hline No. dead during treatment & 0 & $2^{\mathrm{a}}$ & $1^{\mathrm{a}}$ & 0 & $1^{\mathrm{a}}$ & $2^{\mathrm{b}}$ & 0 & 0 & $1^{\mathrm{b}}$ & $1^{\mathrm{b}}$ & 0 & 0 \\
\hline No. moribund and sacrificed & 0 & $2^{\mathrm{a}}$ & $1^{\mathrm{a}}$ & 0 & $1^{\mathrm{a}}$ & $2^{b}$ & 0 & 0 & $1^{\mathrm{b}}$ & $1^{\mathrm{b}}$ & 0 & 0 \\
\hline No. finally sacrificed & 10 & 8 & 9 & 10 & 9 & 8 & 10 & 10 & 9 & 9 & 10 & 10 \\
\hline No. examined for gross pathology & 10 & 10 & 10 & 10 & 10 & 10 & 10 & 10 & 10 & 10 & 10 & 10 \\
\hline No. showing gross pathology & 0 & 0 & 0 & 0 & 0 & 0 & 0 & 0 & 0 & 0 & 0 & 0 \\
\hline Visceral organ pathology & 0 & 0 & 0 & 0 & 0 & 0 & 0 & 0 & 0 & 0 & 0 & 0 \\
\hline
\end{tabular}

a Animals died during the blood drawing on 60 day of post exposure.

b Animals found dead during the treatment after 4 therapeutic dose (3.5\%).

phoid hyperplasia-VC, DRV $(2 \times)$ and DRV $(10 \times)$; injection site abscess-VC; intestinal mucosa associated lymphoid tissue hyperplasia—DRV $(1 \times), \mathrm{CRV}(1 \times), \mathrm{DRV}(2 \times)$ and DRV $(10 \times))$. However, the changes observed did not appear to be related to the treatment since there were no dose related changes and were also observed in the vehicle controls and were not statistically significant.

\section{Discussion}

The DRV, an eukaryotic expression plasmid encoding rabies virus glycoprotein consisting of kanamycin resistance gene as the antibiotic selection marker induces virus neutralizing antibodies in mice and monkeys and protects mice partially against intracerebral and peripheral rabies virus challenge [5,3,4]. Abhayrab, a purified vero cell-derived human anti-rabies vaccine (PVRV) consisting of inactivated rabies virus induces high levels of virus neutralizing antibodies in humans [19]. Addition of a small quantity of PVRV to DRV was shown to dramatically enhance the potency of the former leading to the development of a novel combination anti-rabies vaccine formulation or CRV [4]. In order to develop CRV as a low cost human anti-rabies vaccine, it was necessary to carry out safety and pre-clinical toxicity studies in appropriate animal species. In order to promote any drug or vaccine for clinical use, the primary requirement in preclinical toxicology studies is the species selection. Selection of the species varies with the nature of the compound under test [23] and the safety evaluation of biological products which include biotech manufactured substances need relevant species having relative affinity, distribution of receptors for the intended clinical product with appropriate immunological response [23]. In the present study, the rodent species (Swiss Albino mice) had been selected which is not only as per regulatory guidelines, but also based on the efficacy studies $[5,3,4]$. These studies have successfully demonstrated the induction of higher anamnestic antibody response in Swiss, Balb C mice and cattle after administration of CRV vaccine (DRV + cell culture vaccine) thus confirming and supporting our selection of the appropriate rodent species.
Conventional toxicology procedures support the hypothesis on safety of compound for clinical use, only if it does not indicate any adverse reaction, after administration of 10 times of intended therapeutic dose [16]. The selection of dosage and duration is based on the principles of toxicology studies and its vaccination potential. As per the guidelines, monitoring of various parameters includes physical, physiological, specially allergic, clinical, hematological, biochemical and histopathological studies.

In the present study, the data on acute, sub-chronic and chronic toxicity test are in agreement with the sound scientific reports published for various products. Since the product is a DNA based vaccine, and is an immuno-protective agent, special attention on evaluation of the immune toxic effects was based on tier-I tests $([2,21,22])$. The initial tier-I screen for immunotoxic effects are based on routine hematological, serological and histopathological findings. In case of any abnormality, the tier-II immune parameters need to be monitored. In the present investigation, the results, specially total and differential blood cell counts, serum protein, albumin, organ weights, body weights were found to be in normal ranges even at the highest concentration of post exposure for 17-18 weeks.

The detailed investigations carried out in the present study are according to the updated guidelines as well as report of Cockerell et al. [6] (specifically on data related to histopathological and other toxicological findings). The routine parameters specially food intake, body weight, physical, physiological and clinical parameters did not show any significant changes even after the exposure of the animals with 5 and 10 times the recommended therapeutic dose. The biochemical and hematological profile also were normal. In addition to these, tier-I tests (immunotoxicology) were also carried out. There was no evidence of histopathological changes in spleen, thymus, lymph nodes and bone marrow in animals exposed to ten times the therapeutic dose, suggesting the safety of the test material. Also there was no evidence of infection/tumor, particularly of lymphoid tissues in the animals exposed to DRV or CRV, even after 120 days of post exposure at $10 \times$ dose. These results thus, suggest no evidence of immunotoxicity due to DRV and CRV. The data generated in the present study from Indian laboratories is 
according to the guidelines proposed by the Department of Biotechnology, New Delhi [2] to evaluate the safety trials of indigenous biotech products. Since the DNA construct is the major inducer of immunity in the present anti-rabies vaccine, it is essential to examine its immunotoxicological effects like induction of antinuclear antibodies/autoimmunity. Though, first generation cross between New zealand white and black mice has been suggested as a suitable model for assessing autoimmunity, we were not able to carry out these studies due to non-availability of the above strains. However, induction of anti nuclear antibodies and double stranded DNA following DRV/CRV immunization were evaluated in another study using non human primates which were used as a second species as is mandatory according to the regulatory guidelines (to be published).

This data adds information to the existing data on various other DNA products to be used as preventive/therapeutic agents in clinical conditions. The present study conducted in Indian laboratories is an attempt to address the importance of developing preclinical toxicity data on a case by case basis with special reference to biotech products.

\section{Acknowledgements}

We sincerely acknowledge the technical assistance rendered by Mr. P. Nagesh Babu, Mrs. P. Madhavi, Mr. Ramachandra Rao, Mrs. Shailaja, Mr. Kiran Kumar, Mrs. B. Tulja, Mr. Y. Bala Narayana and Mr. Giribabu in different aspects of the work.

\section{References}

[1] Bahloul C, Jacob Y, Tordo N, Perrin P. DNA-based immunization for exploring the enlargement of immunological cross-reactivity against the lyssaviruses. Vaccine 1998;16:417-25.

[2] Biotech Consortium India Limited and Department of Biotechnology, GOI, National Consultation on Biosafety aspects related to genetically modified organisms, India, 2004.

[3] Biswas S, Kalanidhi AP, Ashok MS, Raddy GS, Srinivasan VA, Rangarajan PN. Evaluation of rabies virus neutralizing antibody titres induced by intramuscular inoculation of rabies DNA vaccine in mice and Bonnet monkeys. Indian J Exp Biol 2001;39:533-6.

[4] Biswas S, Reddy GS, Srinivasan VA, Rangarajan PN. Preexposure efficacy of a Novel Combination DNA and inactivated rabies virus vaccine. Human Gene Ther 2001;12:1917-22.

[5] Biswas S, Ashok MS, Reddy GS, Srinivasan VA, Rangarajan PN. Evaluation of the protective efficacy of a rabies DNA vaccine in mice using an intracerebral challenge model. Curr Sci 1999;76: 1012-6.
[6] Cockerell GL, Aaron CS, Moe JB. Commentary: redesigning the preclinical paradigm: the role of pathology and toxicology in supporting discovery research. Toxicol Pathol 1999;27:477-8.

[7] Drug and Cosmetics (Eighth Amendment Rules 1988 of Drug and Cosmetic Act)) Act, of 1940. Schedule Y Govt. of India. Drug Controller General of India; Requirement and guidelines of clinical trials or import and manufacture of new drug, 2002.

[8] FDA, 1997a. Guidelines for industry and reviews repeal of section 507 of Federal Food, Drug and Cosmetic Act, USA (http://www.fda.gov/cder/guidance/index.htm).

[9] FDA, 1997b. Points to consider in the manufacturing and testing of monoclonal antibody; products for human use. Federal Food, Drug and Cosmetic Act, USA.

[10] Fishbein DB, Robinson LE, Rabies N. Engl J Med 1993;329:1632-8.

[11] Fu ZF. Rabies and rabies research: past, present and future. Vaccine 1997; 15:S20-4.

[12] Genton B, Al yaman F, Betuela I, Anders RF, Saul A, Baea K, et al. Safety and immunogenicity of a three component blood stage malaria vaccine (MSP1, MSP2 RESA) against plasmodium falciparum in Papua New Guinea children. Vaccine 2003;22:30-41.

[13] Horn NA, Meek JA, Budahazi G, Marquet M. Cancer gene therapy using plasmid DNA: purification of DNA for human clinical trials. Hum Gene Ther 1995;6(5):565-73.

[14] ICH, International Conference on Harmonization, Safety steps 4/S documents Buffalo Grove 12, Interpharma press, 1997.

[15] ICH, Preclinical safety evaluation of biotechnology derived pharmaceuticals, 1997.

[16] Paget, GE, Barnes, JM. Evaluation of drug activities. In: Laurence DR, Bacharach AL., editors. Pharmacometrics, vol. 1, London, Academic Press; 1964.

[17] Petricciani JC. Recombinant DNA vaccines and therapeutics. Lancet 1993;342:1067-8.

[18] Ray NB, Ewalt LC, Lodmell DL. Nanogram quantities of plasmid DNA encoding the rabies virus glycoprotein protect mice against lethal rabies virus infection. Vaccine 1997;15:892-5.

[19] Sampath G, Reddy SV, Rao ML, Rao YU, Palaniappan C. An immunogenicity study of a newly introduced purified Vero cell rabies vaccine (Abhayrab) manufactured in India. Vaccine 2005;23(7):897-900.

[20] Tullu MS, Rodrigues S, Muranjan MN, Bavdekar SB, Kamat JR, Hira PR. Neurological complications of rabies vaccines. Indian Pediatr 2003;40:150-4.

[21] US Department of Health and Human Sciences, FDA (CDER) (2001) Immunotoxicology Evaluation of Investigational New Drug G.3010 DFT. Doc, http://www.fda.gov/cder/guidance/index.htm.

[22] Warner GL, Haggerty HG. Immunotoxicology of recombinant DNA derived therapeutic proteins. In: Comprehensive Toxicology, vol. 5, Lawrence DA, editor. Toxicology of the Immune System. NY, USA: New York State Dept. of Health; 1997.

[23] Weissnger J. Preclinical pharmacology and toxicology of haemopoitic factors. Biotech Advances 1989;7:387-99.

[24] Xiang Z, Ertl HCJ. Manipulation of the immune response to a plasmid-encoded viral antigen by coinoculation with plasmids expressing cytokines. Immunity 1995;2:129-35.

[25] Xiang ZQ, Spitalnik S, Tran M, Cheng J, Ertl HCJ. Vaccination with a plasmid vector carrying the rabies virus glycoprotein gene induces protective immunity against rabies virus. Virology 1994;199:132-40. 